\title{
Influence of the Duct Shape on the Q-Factor of a Photoacoustic Helmholtz Resonator
}

\author{
M. Suchenek
}

Received: 15 April 2010 / Accepted: 17 May 2010 / Published online: 12 June 2010

(C) The Author(s) 2010. This article is published with open access at Springerlink.com

\begin{abstract}
The use of a Helmholtz resonator as a photoacoustic cell has many advantages. Its main drawback in high-sensitivity photoacoustic measurements is the relatively low values of Q-factors of such cells. One of the important loss sources which limit the quality factor of photoacoustic Helmholtz resonators is the rapid change of the cross section at the cavity-duct boundaries. This paper presents a design of a cell in which the shape of the duct was modified in such a way that the cross-section change is more gradual. A presented comparison of measurements of the Q-factors shows clearly that the Q-factor of the cell can be improved by almost a factor of two by such a modification of the duct shape.
\end{abstract}

Keywords Acoustic resonator $\cdot$ Helmholtz resonator $\cdot$ Photoacoustic cell design

\section{Introduction}

Helmholtz resonators are often used in photoacoustic measurements, and have several advantages in such applications. The most important is probably that the total volume of a photoacoustic Helmholtz cell can be kept small - at the level of a few $\mathrm{cm}^{3}$ or less-even if a $2.54 \mathrm{~cm}$ ( $1 \mathrm{in}$.) microphone is used. Simultaneously, the resonance frequency of such a cell can be still around $1 \mathrm{kHz}$ or even lower. These features are very valuable, because the resonance can be used for the purpose of signal amplification, and due to the $Q / f \cong V$ dependence, low values of the cell volume and the light beam modulation frequency would result in a higher level of the photoacoustic signal. Unfortunately, the quality factors Q of photoacoustic Helmholtz cells are not

\footnotetext{
M. Suchenek $(\varangle)$

Institute of Electronic Systems, Warsaw University of Technology, Nowowiejska 15/19,

00-665 Warsaw, Poland

e-mail: M.Suchenek@elka.pw.edu.pl
} 
very high $[1,2]$. The aim of this study was to investigate if it is possible to noticeably increase the Q-factor of such a resonator by means of a simple modification of its duct shape.

\section{Modified Design of Helmholtz Resonator}

A photoacoustic Helmholtz cell consists of two cavities connected with a duct (Fig. 1). Such an acoustic structure can be modeled in many ways, but the most common is an approach of acousto-electrical analogies, in which the properties of every acoustical component are described by means of appropriately selected electrical counterparts. In particular, cavities are modeled by capacitors, while the duct is modeled by a series connection of an inductance with a resistance or by a transmission line (Fig. 2). Values of these components can be defined differently, but in all such models of photoacoustic or small acoustic Helmholtz resonators, the dominant losses are introduced by the duct connecting the cavities [3-8]. Certainly, the value of these losses depends on the duct dimensions. Hence, by appropriate selection of the duct dimensions, it is possible to reduce losses within the resonator, which results in an increase of its Q-factor. Obviously, not only the dimensions, but also the shape of the duct has an influence on the Q-factor of the cell. There are papers which suggest that probably one of the most important loss sources involves flow disturbances due to the rapid change of a cross section at the duct-cavity boundary [9]. A connection which results in smaller losses can be a horn [10,11]. A horn reduces reflectance losses in comparison to a design with a rapid change of the cross section between the duct and cavity. There are similar practical solutions in acoustics [12] and theoretical solutions in photoacoustics [13]. However, the molding of a horn, especially for the case of small dimensions, which are typical for photoacoustic Helmholtz resonators, is not easy. Much easier in physical implementation is a cone profile. As a result of such a modification, a Helmholtz resonator would have a structure as given in Fig. 3.

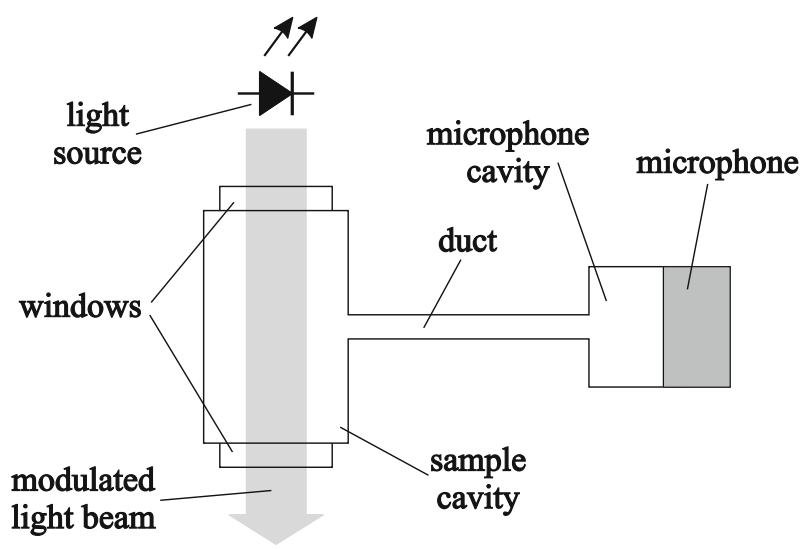

Fig. 1 Sketch of a photoacoustic Helmholtz resonator 

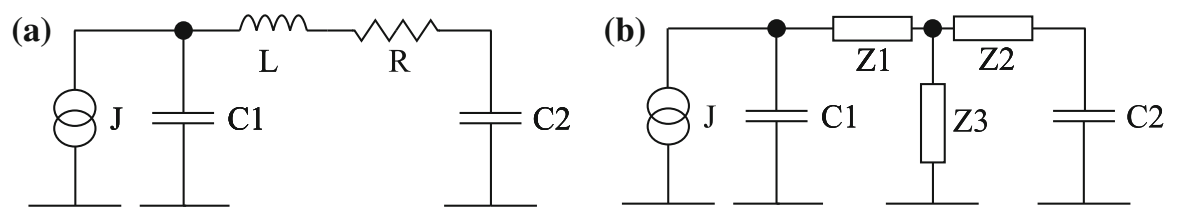

Fig. 2 Helmholtz resonator models: (a) model with lumped elements and (b) model with transmission line

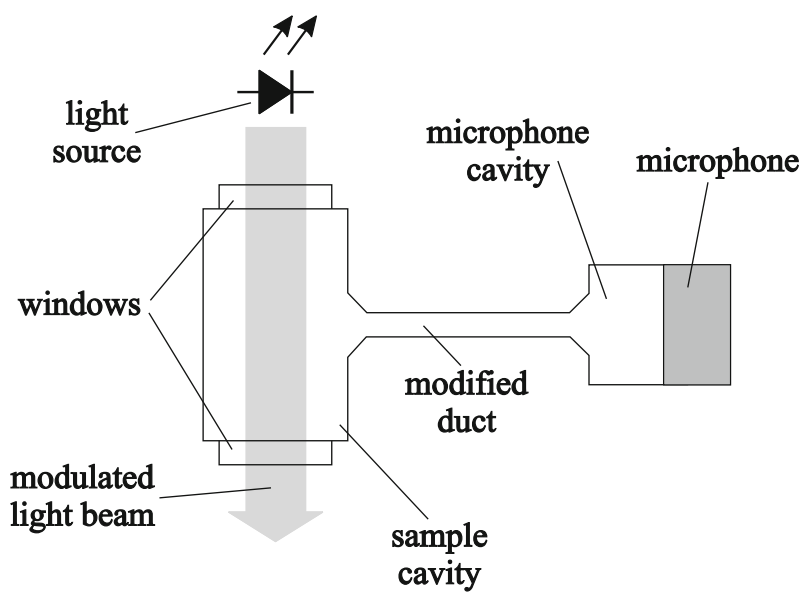

Fig. 3 Sketch of a modified photoacoustic Helmholtz resonator, in which the duct has conical ends

\section{Measurement Results}

To investigate the influence of the presented modification of a photoacoustic Helmholtz cell on its Q-factor, several cells with different dimensions of the conical ends of the duct were investigated. At first the frequency response of every cell was recorded, and then its Q-factor was calculated. In all, both experimental resonators had cavities of $2 \mathrm{~cm}^{3}$, while the duct was $10 \mathrm{~mm}$ long (in total, i.e., including the conical ends) and with $2 \mathrm{~mm}$ in diameter. To reduce errors that would result from the change of other dimensions of the cell, the measured resonator was designed in such a way, that both cavities were the same in all tests and only the middle part, containing the duct, was changed (Fig. 4).

In the first experiment, the length of both conical segments of the duct was changed from $0.0 \mathrm{~mm}$ to $4.5 \mathrm{~mm}$ each. A resonator with no conical segments $(0.0 \mathrm{~mm})$ was used as a reference. In this experiment, the angle between the surface of the conical segments and the duct axis was $33^{\circ}$ in all cases. Values of the Q-factors extracted from the measurements of these cells with all the lines approximating the measured data are presented in Figs. 5 and 6.

It is clearly visible from the figures that an increase of the conical segment length results in an increase of the Q-factor as well as the resonance frequency. Both relationships seem to be close to linear. However, the most important result is that the 


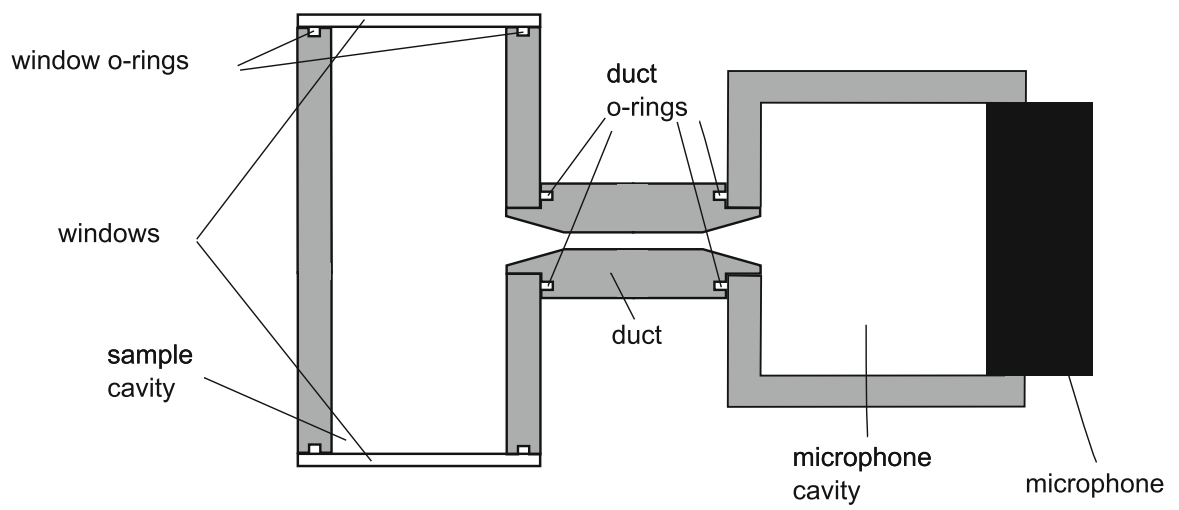

Fig. 4 Mechanical design of modified Helmholtz resonator

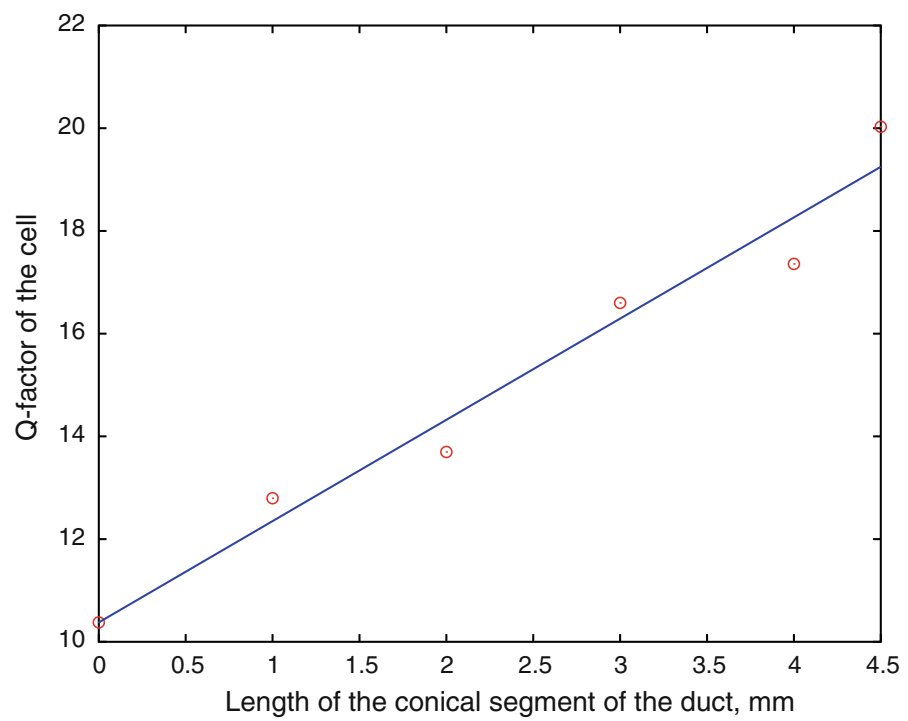

Fig. 5 Influence of the conical duct segment length on the Q-factor of the cell

Q-factor of the cell can be substantially higher if the duct is equipped with conical ends. In particular, for the maximum investigated conical segment length of $4.5 \mathrm{~mm}$ on each side of the duct, the Q-factor was $92.9 \%$ higher in comparison to the nonmodified duct.

In the second experiment, the influence of the angle between the conical segment surface and the duct axis on the Q-factor and resonance frequency of the cell was studied. For this purpose, ducts with six different values of the angle were used: $0^{\circ}$, $5^{\circ}, 12^{\circ}, 22^{\circ}, 32^{\circ}$, and $42^{\circ}$. In all these cases, the conical segment length was $3 \mathrm{~mm}$. Measurement results of these cells are given in Figs. 7 and 8.

The second experiment showed that both the Q-factor and resonance frequency increase with the angle between the conical segment surface and the duct axis. For the investigated cells, the maximum gain of the Q-factor in comparison to the nonmodified 


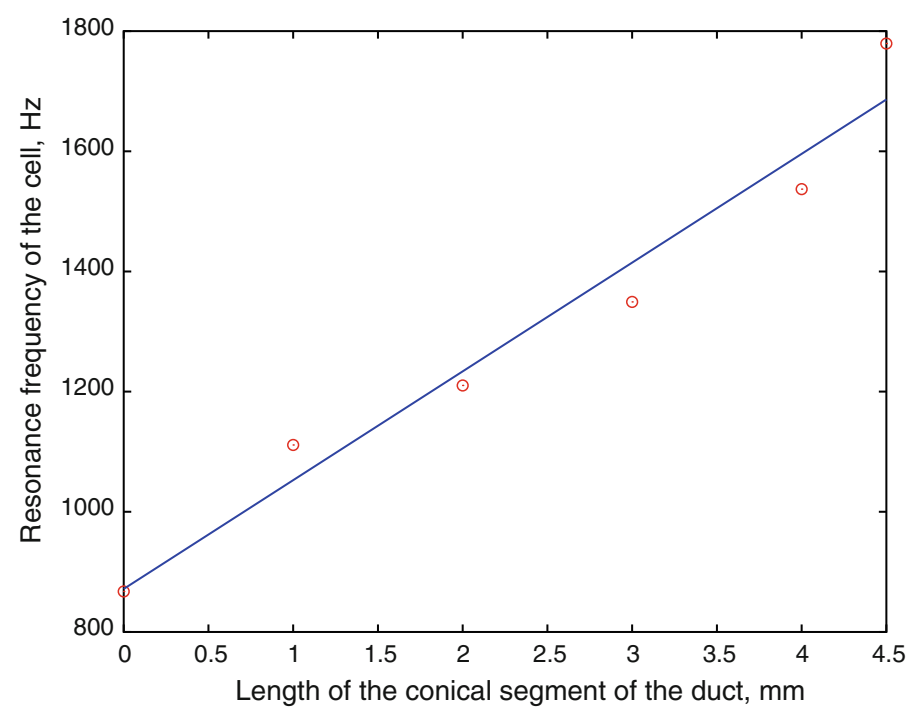

Fig. 6 Influence of the conical duct segment length on the resonance frequency of the cell

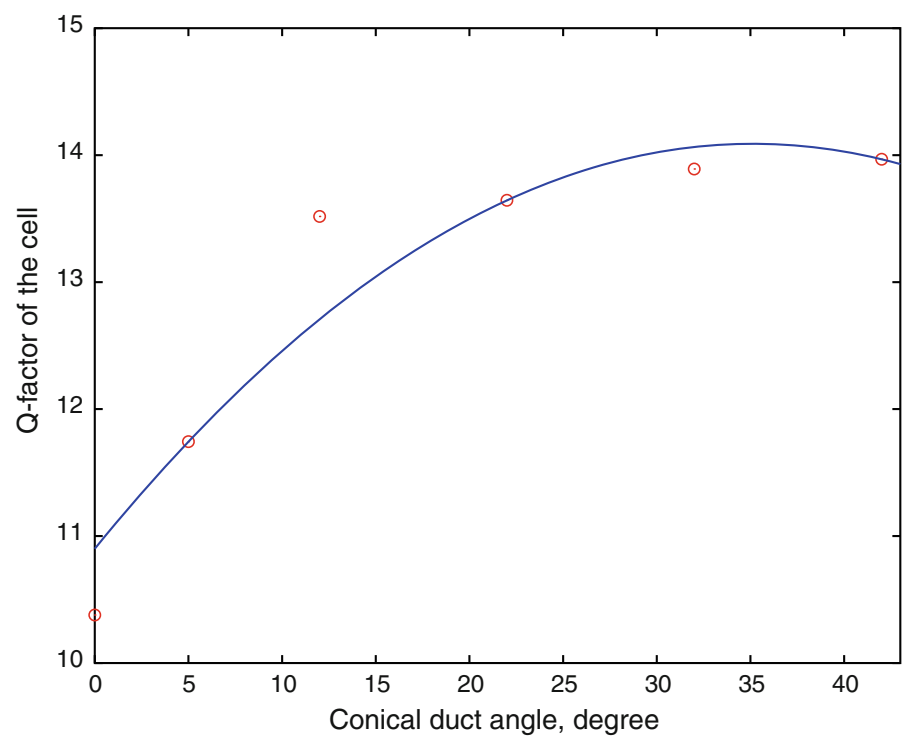

Fig. 7 Influence of the angle between the conical segment surface and the duct axis on the Q-factor of the cell

duct shape was $34 \%$, and was obtained at an angle value of $42^{\circ}$. For the same angle, the resonance frequency increase was also maximal and equal to $35 \%$. It should be noted that the Q-factor versus conical angle relationship is strongly nonlinear. At low values of the angle, even a slight increase results in a substantial gain of the Q-factor. In particular, an increase of the angle from $0^{\circ}$ to $12^{\circ}$ increases the Q-factor by over 


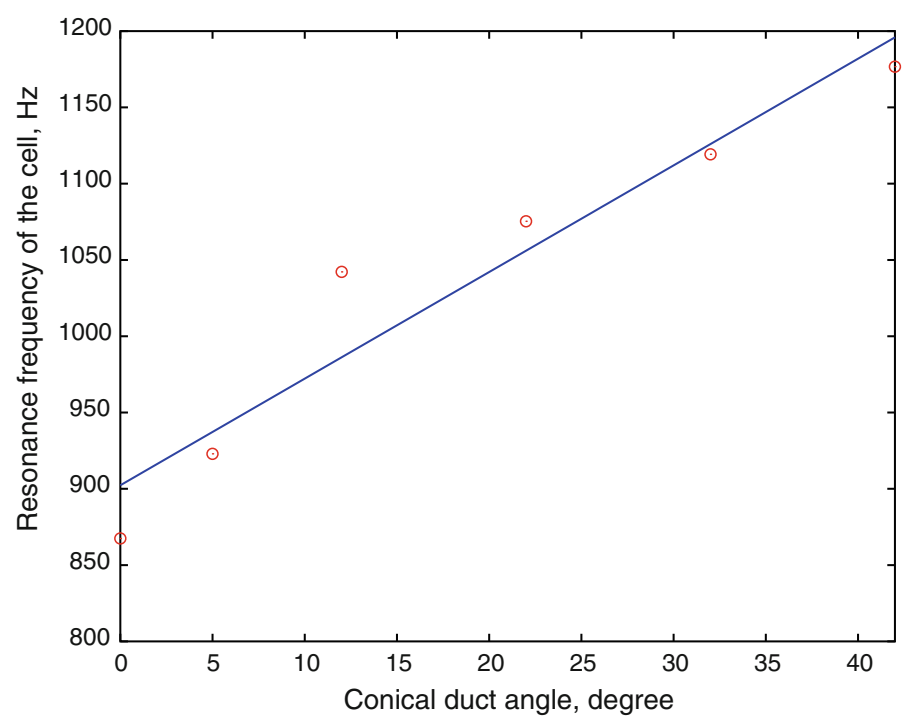

Fig. 8 Influence of the angle between the conical segment surface and the duct axis on the resonance frequency of the cell

$30 \%$, while a further change of the angle from $12^{\circ}$ to $42^{\circ}$ leads to less than $5 \%$ of additional gain of the Q-factor.

\section{Conclusions}

The present results of investigations of photoacoustic Helmholtz cells with a modified shape of the duct show clearly that the use of conical segments at the duct ends may significantly increase the Q-factor of the cell without introducing too many difficulties in its mechanical design and implementation. The maximum gain of the Q-factor in the tested cell configurations in comparison to the reference cell with a nonmodified duct was nearly $100 \%$. Cells with the modified duct had also a higher resonance frequency. Although the presented results are only preliminary and a general description of the influence of the conical duct ends on cell characteristics requires further investigations, it is already clear that such modifications may be particularly advantageous when applied in photoacoustic Helmholtz resonators.

Open Access This article is distributed under the terms of the Creative Commons Attribution Noncommercial License which permits any noncommercial use, distribution, and reproduction in any medium, provided the original author(s) and source are credited.

\section{References}

1. V.P. Zharov, V.S. Letokhov, Laser Optoacoustic Spectroscopy (Springer-Verlag, Germany, 1986), p. 98

2. R.S. Quimby, P.M. Selzer, W.M. Yen, Appl. Opt. 16, 2630 (1977) 
3. R. Kastle, M.W. Sigrist, Appl. Phys. B 63, 389 (1996)

4. M. Mattiello, M. Nikles, S. Schilt, L. Thevenaz, A. Salhi, D. Bart, Y. Rouillard, R. Werner, J. Koeth, Spectrochim. Acta A 63, 952 (2006)

5. O. Nordhaus, J. Pelzl, Appl. Phys. A 25, 221 (1981)

6. J. Pelzl, K. Klein, O. Nordhaus, Appl. Opt. 21, 94 (1982)

7. M. Suchenek, Proc. SPIE 6937, 693711 (2007)

8. V. Zeninari, B. Parvitte, D. Courtois, V.A. Kapitanov, Yu.N. Ponomarev, Infrared Phys. Technol. 44, 253 (2003)

9. T. Starecki, J. Acoust. Soc. Am. 122, 2118 (2007)

10. L.L. Beranek, Acoustics (Acoustical Society of America/American Institute of Physics, New York, 1960), p. 141

11. W. Moon, Y.G. Kim, J. Sound Vib. 259, 779 (2003)

12. S.K. Tang, J. Sound Vib. 279, 1085 (2005)

13. B. Baumann, B. Kost, M. Wolff, H. Groninga, T, Blöß, S. Knickrehm, Proc. Eur. COMSOL Conf. (2007), p. 1 